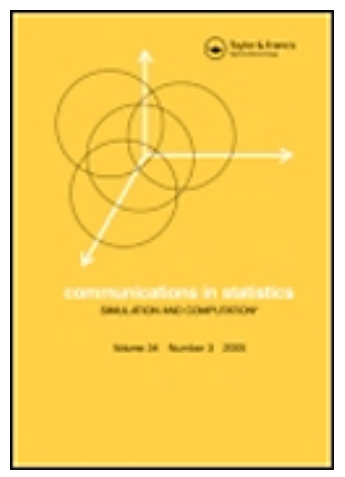

\title{
New prediction interval and band in the nonlinear regression model: Application to predictive modelling in food science
}

\begin{tabular}{|c|c|}
\hline Journal: & Communications in Statistics - Simulation and Computation \\
\hline Manuscript ID: & LSSP-2007-0136.R2 \\
\hline Manuscript Type: & Original Paper \\
\hline $\begin{array}{r}\text { Date Submitted by the } \\
\text { Author: }\end{array}$ & 14-Oct-2009 \\
\hline Complete List of Authors: & $\begin{array}{l}\text { GAUCHI, Jean-Pierre; INRA, MIA(UR341) } \\
\text { VILA, Jean-Pierre; INRA, LASB } \\
\text { COROLLER, Louis; UBO, LUMAQ }\end{array}$ \\
\hline Keywords: & $\begin{array}{l}\text { Prediction interval, Prediction band, Nonlinear regression, } \\
\text { Parametric confidence region, Predictive modelling, Food Science }\end{array}$ \\
\hline Abstract: & $\begin{array}{l}\text { We propose a new prediction interval and band for the nonlinear } \\
\text { regression model. The construction principle of this interval and } \\
\text { band is based on an exact confidence region for parameters of the } \\
\text { nonlinear regression model. This region, fully described in Vila \& } \\
\text { Gauchi (2007), provides a rigorous justification for the new } \\
\text { prediction interval and band that we propose. This new band is then } \\
\text { compared to the classical bands, and also to the band based on the } \\
\text { bootstrap resampling method. The comparison of these bands is } \\
\text { undertaken with simulated and real data from predictive modelling } \\
\text { in food science. }\end{array}$ \\
\hline \multicolumn{2}{|c|}{$\begin{array}{l}\text { Note: The following files were submitted by the author for peer review, but cannot be converted } \\
\text { to PDF. You must view these files (e.g. movies) online. }\end{array}$} \\
\hline revisedarticle.zip & \\
\hline
\end{tabular}




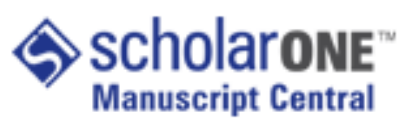


Title:

${ }_{4}$ Authors: Jean-Pierre Gauchi ${ }^{(1) *}$, Jean-Pierre Vila $^{(2)}$, Louis Coroller ${ }^{(3)}$

$6 \quad$ (1) INRA (National Institute of Agronomical Research)

7 Department of Applied Mathematics and Computational Science

$8 \quad$ Unité MIA (UR341MIA); *Corresponding author Application to predictive modelling in foods

\section{Affiliation:}

Domaine de Vilvert, 78352 Jouy-en-Josas, France

(2) INRA (National Institute of Agronomical Research)

Department of Applied Mathematics and Computational Science

UMR Analyse des Systèmes et Biométrie

2, Place P. Viala, 34060 Montpellier, France

(3) Université de Bretagne Occidentale, LUMAQ, Quimper, France

E-mail of corresponding author: Jean-Pierre.Gauchi@jouy.inra.fr

Postal address of corresponding author:

Dr. J.P. Gauchi / INRA / Unité MIA

Domaine de Vilvert

78352 Jouy-en-Josas Cedex, France

New prediction interval and band in the nonlinear regression model: 


\section{${ }_{33} 1$ Introduction}

\section{Abstract:}

This paper is concerned with the proposal of a new prediction interval and band for the nonlinear regression model. The construction principle of this interval and band is based on an exact (the meaning of the term "exact" will be given later) confidence region for parameters of the nonlinear regression model. This region, fully described in Vila \& Gauchi (2007), provides a rigorous justification for the new prediction interval and band that we propose. This new band is then compared to the classical bands (which are asymptotic and thus approximate for small $n$ ), and also to the band based on the bootstrap resampling method. The comparison of these bands is undertaken with simulated and real data from predictive modelling in food science.

Keywords: Prediction interval; Prediction band; Nonlinear regression; Parametric confidence region; Predictive modelling; Food Science.

The question of the construction of prediction intervals $(P I)$ for a single value or a mean and for prediction bands $(P B)$ was extensively studied and solved in the case of linear regression models. The most frequently used solution was developped by Working and Hotelling (1929) and is detailed in Draper and Smith (1981). It leads to the familiar hyperbolic curves surrounding the regression model 
curve. However, several methods are always in competition for nonlinear regression models, and there is no rule for making a definitive choice for a given model. Our aim is therefore to compare four well-known $P I$ and $P B$ on simulated and real data to the new $P I$ and $P B$ we propose, using familiar predictive microbiology models in foods. The first two $P I$ and $P B$ are based on a first-order Taylor approximation of the model analytic form and are called aymptotic $P I$ and $P B$ (see Bates and Watts, 1988). The third $P I$ and $P B$ are based on the bootstrap resampling method (Efron and Tibshirani, 1993). The new PI and PB we propose are based on an exact (see Section 4 for the meaning of the term "exact") confidence parametric region (the $X$-region fully described in Vila \& Gauchi, 2007). The new PI will be referred to as $P I_{X}$ and the new $P B$ as $P B_{X}$.

It is necessary to draw the reader's attention to a crucial point here. We are not actually dealing with genuine prediction regions (note the difference between the words "regions" and "bands") of the model curve. A true prediction region for the model curve is a region where there is a fixed probability of finding this whole model curve completely inside this region. In fact, this question has not yet been totally solved because a genuine region can be too large and of no use to the practitioner. Thus, an approach based on a genuine prediction region is not always a relevant approach. Instead, we prefer to propose a $P B$ made of joint adjacent prediction intervals. The global predictive capability will therefore be 
quantified by the band area. The rest of the article is organized as follows. Section

2 presents some useful notations for the reader. Section 3 provides information on asymptotic and bootstrap bands. Section 4 gives details on the new $P I_{X}$ and $P B_{X}$ we propose. Section 5 is devoted to the comparison of the four bands in the food science predictive modelling field; and Section 6 is the conclusion.

\section{Notations}

Let us consider the standard parametric nonlinear regression model

$$
y_{i}=\eta\left(x_{i}, \theta^{*}\right)+\varepsilon_{i} \quad ; \quad i=1, \ldots, n
$$

with the following notations: $(a) y_{i}$ is the $i^{\text {th }}$ observation of the response (the corresponding $n \times 1$ vector of the $y_{i}$ is referred to as $\left.y\right)$; $(b) x_{i}$ is an $m \times 1$ vector a support point - with component $x_{i l}, l=1, \ldots, m$, and $x_{i} \in \Xi \subset \mathbb{R}^{m}$, where $\Xi$, a compact subset of $\mathbb{R}^{m}$, is the experimental domain. For the sake of briefness, we will refer to the $n \times m$ matrix $X$ as the whole set of the $m$ explanatory variables set at $n$ levels; $(c)$ We assume that the errors $\varepsilon_{i}$, components of the $n \times 1$ vector $\varepsilon$, are independent and normally distributed; they have the same variance $\sigma^{2}$ (generally unknown). Then, $\varepsilon \sim \mathcal{N}(0, \Sigma)$, where $\Sigma=\sigma^{2} I_{n}\left(I_{n}\right.$ is the $n \times n$ identity matrix $) ;(d)$ $\theta^{*}$ is the $p \times 1$ vector of the unknown $p$ parameters to be estimated, $\theta^{*} \in \Theta \subset \mathbb{R}^{p}$, where $\Theta$ is the parametric domain, a compact subset of $\mathbb{R}^{p} ;(e) \eta($.$) is a continuous$ 
76 real-valued scalar function of $x$ defined on $\Xi \times \Theta$, twice differentiable with respect

77 to the parameters and the variables; we will simply refer to the corresponding

${ }_{78} n \times 1$ vector as $\eta$ or $\eta_{\theta}$ (function of the $\theta$ variable); $(f) n$ is the total number of

79 observations $y_{i}$.

80 We used the ordinary nonlinear least squares estimate $\hat{\theta}$ as an estimate of $\theta^{*}$

$$
\hat{\theta}=\underset{\theta \epsilon \Theta}{\operatorname{Argmin}}\left\{\left(y-\eta_{\theta}\right)^{T} \Sigma^{-1}\left(y-\eta_{\theta}\right)\right\}=\operatorname{Arg} \min _{\theta \in \Theta}\left\|y-\eta_{\theta}\right\|_{\Sigma}^{2}
$$

${ }_{81}$ We also considered the Fisher information matrix $M_{F}\left(X, \theta^{*}\right)$

$$
M_{F}\left(X, \theta^{*}\right)=\sigma^{-2} J_{X, \theta^{*}}^{T} J_{X, \theta^{*}}
$$

82 where $J_{X, \theta^{*}}$ is the $n \times p$ Jacobian matrix, with the $j^{\text {th }}$ column consisting of the $n$

83 components of $\partial \eta\left(X, \theta^{*}\right) / \partial \theta_{j}^{*}, j=1, \ldots, p$.

84 A model prediction at any point $x_{i}$ is defined as

$$
\hat{y}_{i}=\eta\left(x_{i}, \hat{\theta}\right)
$$

${ }_{85}$ For further use, we also define the $(1 \times p)$-vector, a row of $J_{X, \theta}$, as:

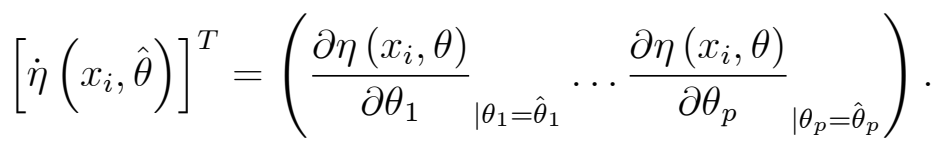




\section{Classical $P I$ and $P B$}

Thus, the first step is to construct a $P I$ for $y_{0}$ (located on $\left.x_{0}\right)$ defined as:

$$
P I\left(y_{0}, x_{0}, \alpha\right)=\left[L\left(\hat{y}_{0}, x_{0}, \alpha\right) ; U\left(\hat{y}_{0}, x_{0}, \alpha\right)\right]
$$

96

As reported by Khorasani \& Milliken (1982), the problem of constructing a prediction band around a regression model can be viewed as a problem of simultaneously constructing an infinite set of confidence intervals. Let us assume that $E\left(y_{0}\right)=$ $\eta\left(x_{0}, \theta^{*}\right)$ is the mean of some $y_{0}$, and that $\hat{y}_{0}=\hat{E}\left(y_{0}\right)=\eta\left(x_{0}, \hat{\theta}\right)$, the mean prediction, is its estimation. We are interested in determining a $P I$ for $y_{0}$, followed by a $P B$ for the regression model. The objective will be fully satisfied if the confidence level of the $P I s$ and that of the $P B s$ is as close as possible to $1-\alpha$ where $\alpha$ is a first-kind error.

where $L\left(\hat{y}_{0}, x_{0}, \alpha\right)$ is the lower bound of the interval, and $U\left(\hat{y}_{0}, x_{0}, \alpha\right)$ is the upper bound of the interval.

The second step is then to determine a $P B$ over $\Xi$. It should be observed that if $\Xi \subset R^{m}$, with $m=1$, we then consider a band, but if $m \geq 2$, we will refer to it as a prediction region $(P R)$, different from a genuine prediction region (see the crucial point in the introduction). The upper curve (or the $m$-dimensional

\subsection{Principle}


where

$$
\hat{T}=\frac{\hat{y}_{0}-y_{0}}{h_{x_{0}}^{1 / 2}(\hat{\theta})}
$$


and where

$$
\hat{\sigma}^{2}=\frac{1}{n-p} \sum_{i=1}^{n}\left(y_{i}-\hat{y}_{i}\right)^{2}
$$

Classical asymptotic theory (Gallant, 1987) tells us that the limiting distribution (when $n$ tends to infinity) of (6) is a centered normal distribution $\mathcal{N}(0,1)$ where $h_{x_{0}}^{1 / 2}(\hat{\theta})$ is an estimate of the standard error of $\hat{y}_{0}$. However, for small fixed $n$, the true distribution of $\hat{T}$ is not a normal one and is not known. Nevertheless, we have somewhat accurate approximations of the true distribution when $n$ is not infinite, which lead to confidence interval approximations. Typically, on the basis of Gallant (1987), we have:

$$
P I_{t}\left(y_{0}, x_{0}, \alpha\right)=\left[\hat{y}_{0}-t_{n-p ; 1-\alpha / 2} h_{x_{0}}^{1 / 2} ; \hat{y}_{0}+t_{n-p ; 1-\alpha / 2} h_{x_{0}}^{1 / 2}\right]
$$

where $t_{n-p ; 1-\alpha / 2}$ is the $(1-\alpha / 2)$ - percentile for the Student distribution with $n-p$ degrees of freedom. The whole set of the joint $P I_{t} s$ leads to the $P B_{t}$.

On the basis of Bates \& Watts (1988), we can use a good alternative defined as:

$$
P I_{F}\left(y_{0}, x_{0}, \alpha\right)=\left[\hat{y}_{0}-\left(h_{x_{0}} p \mathcal{F}_{p, n-p ; \alpha}\right)^{1 / 2} ; \hat{y}_{0}+\left(h_{x_{0}} p \mathcal{F}_{p, n-p ; \alpha}\right)^{1 / 2}\right]
$$

where $\mathcal{F}_{p, n-p ; \alpha}$ is the upper $\alpha$-percentile for the Fisher-Snedecor distribution with $p$ and $n-p$ degrees of freedom. This interval definition leads to a so-called 
silmutaneous prediction band $P B_{F}$.

These methods are called asymptotic in the nonlinear case because it has been proven (see Gallant, 1987) that $P I_{t}$ and $P I_{F}$ tend to an exact $P I$ as $n \rightarrow \infty$. In this case, the word "exact" means that the coverage probability of $y_{0}$ is exactly equal to $1-\alpha$. For a fixed $n$, particularly if $n$ is small, $P I_{t}$ and $P I_{F}$ are only approximate, and their covering probabilities are not equal to $1-\alpha$, and are close to $1-\alpha$ if $n$ is large enough.

\subsection{Interval and band based on the bootstrap method}

This method is based on the famous resampling method called the bootstrap method (Efron and Tibshirani, 1993). Many statistics can be estimated with this method, particularly the construction of confidence intervals, as clearly described in Huet et al. (2004, page 138). We only give the results here:

$$
P I_{B}\left(y_{0}, x_{0}, \alpha\right)=\left[\hat{y}_{0}-b_{1-\alpha / 2} h_{x_{0}}^{1 / 2}(\hat{\theta}) ; \hat{y}_{0}-b_{\alpha / 2} h_{x_{0}}^{1 / 2}(\hat{\theta})\right]
$$

where $b_{1-\alpha / 2}$ and $b_{\alpha / 2}$ are the $1-\alpha / 2$-percentile and $\alpha / 2$-percentile, respectively, of the bootstrap distribution of $\hat{y}_{0}$. Generally, for a given $n$ (not infinity), it can be assumed that $P B_{B}$ will be narrower than $P B_{t}$ and $P B_{F}$ for a confidence level close to $1-\alpha$. 


\section{A new prediction band}

\subsection{The $X$-region}

Because the $P B_{X}$ is based on an exact confidence region for the parameters of a nonlinear regression model - the $X$-region fully described in Vila and Gauchi (2007) - we have included the following definition for the reader's information:

Definition 1 Let $C\left(\theta^{*}\right)$ be the covering probability of $\theta^{*}$. The confidence region of nominal level $\alpha$ for $\theta^{*}$ is then said to be approximate if $C\left(\theta^{*}\right) \approx 1-\alpha$, asymptotic if $\lim _{n \rightarrow \infty} C\left(\theta^{*}\right)=1-\alpha$, conservative if $C\left(\theta^{*}\right) \geq 1-\alpha$, and exact if $C\left(\theta^{*}\right)=1-\alpha$, $\forall n$.

The $X$-region we used is defined as:

$$
\Re_{X}\left(\theta^{*}, y\right)=\left\{\theta \in \Theta: R_{X}(\theta) \leq \mathcal{F}_{p, \nu ; \alpha}\right\}
$$

where $R_{X}(\theta)=\left(y-\eta_{\theta}\right)^{T} P_{\theta}\left(y-\eta_{\theta}\right) / p s_{\nu}^{2}$ with $P_{\theta}=J_{X, \theta}\left(J_{X, \theta}^{T} J_{X, \theta}\right)^{-1} J_{X, \theta}^{T}$, the usual projector onto the plane, tangent at $\eta_{\theta}$, on the model response surface (see Bates and Watts, 1988, p.36, for a clear presentation), and $s_{\nu}^{2}$ an estimate of $\sigma^{2}$, based on $\nu$ degrees of freedom (d.o.f.). $\mathcal{F}_{p, \nu ; \alpha}$ is the $\alpha$-percentile of the Fisher-Snedecor distribution $\mathcal{F}_{p, \nu}$ with $p$ and $\nu$ d.o.f. If $s_{\nu}^{2}$ is not available, it will be replaced by the mean square error (and, thus, $\nu=n-p$ in this case) of the regression procedure. A graph of this region is displayed in Vila and Gauchi (2007). It may look very 
different from the ellipsoidal confidence region often used as an approximative region.

We would like to remind the reader here of some important properties of the $X$-region (Vila and Gauchi, 2007): the $X$-region does not depend on intrinsic and parametric nonlinearities of the model $\eta($.$) , and the true \Theta$ is taken into account in its construction. These properties are enhanced if $n$ is small and $\sigma$ is large. Consequently, the $P B_{X}$ will benefit from these properties of the $X$-region, to the contrary of the preceding $P B s$. Indeed, we know that these two conditions make the usual first-order approximation involved in $P I_{t}$ and $P I_{F}$ inadequate.

\subsection{The $P B_{X}$}

We can now construct the $P B_{X}$. This band is formed by joining $K$ (see Subsection 3.1) adjacent $P I_{X}$. A $P I_{X}$ for $y_{i}$ is then built by means of the four steps of the following simple algorithm based on Monte Carlo samplings:

\section{Step 1}

If $p \leq 3$ : uniformly sample a large number $Q$ of vectors $\tilde{\theta}_{u} \in \Theta, u=1, \ldots, Q$ (typically $Q=1000$ for $p=1, Q=10^{4}$ for $p=2, Q=5 \times 10^{4}$ for $p=3$, since these values are now easily attainable with powerful modern computers). Among the $Q$ vectors $\tilde{\theta}_{u}$, only $Q^{*}$ satisfy the inequality in (12). Thus, the set of these $Q^{*}$ vectors $\tilde{\theta}_{u}$ forms an approximation of the volume of (12). 
If $p>3$ : sample a large number $Q$ of uniform random straight half lines $L_{k} \in \Theta$, $k=1, \ldots, Q$, whose origins are all located at $\theta_{0}$ and whose slopes are random (typically $Q=10^{4}$ for $p=4,5$, or $6, Q=10^{5}$ for $6<p \leq 10$ ). On each random line $L_{k}$, choose a number $q_{k}$ of values $\tilde{\theta}_{u}, u=1, \ldots, q_{k}$, regurlarly spaced on this line. The same stepsize is used for all the lines, and each $q_{k}$ depends on the proximity of $\theta_{0}$ from the border of the $X$-region along the $L_{k}$ line. Finally, only $Q^{*}$ vectors $\tilde{\theta}_{u}$ among the $\sum_{k=1}^{Q} q_{k}$ vectors satisfy the inequality in (12). It should be observed that the sampling used for $p \leq 3$ would be very ineffective here because it is well-known that the random $\tilde{\theta}_{u}$ would be increasingly often located in the corners of $\Theta$ when the dimension of $\Theta$ increases.

Step 2 Choose $x_{i}$ as the place in $\Xi$ where we want to make a prediction.

Step 3 Compute the $Q^{*}$ values $\hat{y}_{i}^{(u)}=\eta\left(x_{i}, \tilde{\theta}_{u}\right), u=1, \ldots, Q^{*}$.

Step 4 Determine the lower bound of the $P I_{X}$ by $L=\min _{u}\left\{\hat{y}_{i}^{(u)}\right\}$, and the upper bound of the $P I_{X}$ by $U=\max _{u}\left\{\hat{y}_{i}^{(u)}\right\}$.

The exact confidence level of this band is not yet clear but we can assume that it tends to $\beta \geq 1-\alpha$ when $Q^{*}$ increases because the function $\eta\left(x_{i}, \theta\right)$ is surjective relative to $\theta$. Consequently, the $P B_{X}$ is a conservative band. 
200

201

202

203

204

206

$$
\begin{aligned}
A & =t+\frac{1}{\mu_{\max }} \ln \left(A_{0}\right) \\
A_{0} & =\exp \left(-\mu_{\max } t\right)+\exp \left(-\mu_{\max } l a g\right)-\exp \left(-\mu_{\max } t-\mu_{\max } l a g\right) \\
B & =1+\frac{\left[\exp \left(\mu_{\max } A\right)\right]-1}{\frac{x_{\max }}{x_{0}}}
\end{aligned}
$$

207 with $\mu_{\max }$ (maximum growth rate), lag (lag time before growth, in hours), $x_{\max }$

${ }_{208}$ (maximal number of bacteria), $x_{0}$ (initial number of bacteria), the four components 
of $\theta^{*}$, and time $t$ (the explanatory factor). $\eta\left(\theta^{*}, t\right)$ is the number of bacteria (in $\log$ units).

\subsubsection{Results with simulated data}

We first present an illustration with the a priori values $\mu_{\max 0}=1 ; \operatorname{lag}_{0}=5$; $x_{\max 0}=10^{8} ; x_{00}=100$, and $\Theta=[0.5 ; 1.5]_{\mu_{\max 0}} \times[2 ; 8]_{\operatorname{lag}_{0}} \times\left[0.5 \times 10^{8} ; 1.5 \times 10^{8}\right]_{x_{\max 0}} \times$ $[50 ; 150]_{x_{00}}$, and $\Xi=[0,50]$. With this information (from the SYMPREVIUS Research Group, see Acknowledgements Section) we simulated ten Gaussian $y_{i}$ values (with standard deviation equal to 0.1 ) at ten equidistant levels of time. We obtained Fig. 1 after computation of $\hat{\theta}=\left(1.18 ; 5.44 ; 1.5 \times 10^{8} ; 50\right)^{T}$ by means of the NLIN procedure of SAS/STAT software.

The figure 1 should be approximately placed here

Fig. 1: The four bands for the Baranyi-Roberts model for one simulated data set (crosses stand for the simulated data). The values of the band areas (calculated with procedures indicated at the end of Subsection 3.1) are: $\mathcal{A}\left(P B_{t}\right)=21$; $\mathcal{A}\left(P B_{F}\right)=36 ; \mathcal{A}\left(P B_{B}\right)=24 ; \mathcal{A}\left(P B_{X}\right)=42$.

For a more relevant comparison, we simulated 1000 data sets, each data set being defined as the pairs $\left\{\left(t_{u}, \tilde{y}_{u}\right) ; u=1, \ldots, 10\right\}$, where the $t_{u}$ were always equal to the ten previous levels. Each random $\tilde{y}_{u}$ was generated by a Gaussian distribution 
$\mathcal{N}\left(y_{i}, \sigma_{S}\right)$ where $y_{i}$ was the previous simulated value. Three values of $\sigma_{S}$ were used: $\sigma_{S}=0.05 ; 0.1 ; 0.5$, these values being typical of real experimental situations. For each $\sigma_{S}$-situation, we provide the simulation results in Table 1.

\begin{tabular}{|c|c|c|c|c|c|}
\hline Areas & $\min$ & $\max$ & $\operatorname{mean}$ & std & cv\% \\
\hline $\mathcal{A}\left(P B_{t}\right)$ & $33 ; 31 ; 38$ & $40 ; 44 ; 91$ & $36 ; 37 ; 63$ & $1.5 ; 3 ; 12$ & $4 ; 8 ; 20$ \\
\hline $\mathcal{A}\left(P B_{F}\right)$ & $57 ; 54 ; 66$ & $69 ; 77 ; 159$ & $63 ; 65 ; 110$ & $2.6 ; 5 ; 21$ & $4 ; 8 ; 19$ \\
\hline $\mathcal{A}\left(P B_{B}\right)$ & $60 ; 60 ; 82$ & $86 ; 125 ; 252$ & $78 ; 97 ; 191$ & $6 ; 18 ; 44$ & $7 ; 18 ; 8$ \\
\hline $\mathcal{A}\left(P B_{X}\right)$ & $62 ; 61 ; 46$ & $64 ; 66 ; 71$ & $63 ; 63 ; 61$ & $0.6 ; 1 ; 5$ & $1 ; 1.7 ; 8$ \\
\hline
\end{tabular}

Table 1: Baranyi-Roberts model: Simulation statistics of the band areas based on 1000 simulated data sets. The figures are given sequentially according to $\sigma_{S}=0.05$; $0.1 ; 0.5$, respectively. For example, for $\sigma_{S}=0.05$, we obtain $\min \left(\mathcal{A}\left(P B_{t}\right)\right)=33$, $\max \left(\mathcal{A}\left(P B_{t}\right)\right)=40, \operatorname{mean}\left(\mathcal{A}\left(P B_{t}\right)\right)=36, \operatorname{std}\left(\mathcal{A}\left(P B_{t}\right)\right)=1.5, \operatorname{cv} \%\left(\mathcal{A}\left(P B_{t}\right)\right)=$ 4 .

\subsubsection{An illustration with real data}

With real data (obtained from the SYMPREVIUS Research Group, see Acknowledgements Section) we obtained Fig. 2 where the estimated model curve is based on $\hat{\mu}_{\max }=0.055 ; \widehat{l a g}=42 ; \hat{x}_{\max }=1.52 \times 10^{8} ; \hat{x}_{0}=4005 ; \Theta=[0.050 ; 0.060]_{\mu_{\max 0}}$ $\times[30 ; 54]_{\text {lago }_{0}} \times[38124759 ; 266660000]_{x_{\max 0}} \times[2715 ; 5294]_{x_{00}}$, and $\Xi=[0 ; 310]$.

The figure 2 should be approximately placed here 
Fig. 2: The four bands for the Baranyi-Roberts model for one real data set (crosses stand for the real data). The values of the band areas are: $\mathcal{A}\left(P B_{t}\right)=70$; $\mathcal{A}\left(P B_{F}\right)=111 ; \mathcal{A}\left(P B_{B}\right)=98 ; \mathcal{A}\left(P B_{X}\right)=166$

\subsubsection{Discussion}

We can see on Fig. 1 that the $P B_{t}$ and $P B_{F}$ bands are totally inadequate when the model appears as a zero-slope straitght line. Indeed, the band is reduced to a single line while the variability of the prediction is not null. On the contrary, the other two bands seem more realistic since their width is not null. Moreover, we can see on Fig. 2 that more data lie outside the $P B_{t}, P B_{F}$ and $P B_{B}$, contrast to the $P B_{X}$. In Table 1, we can observe that the $P B_{X}$ does not appear to be not very sensitive to the change of the simulation $\sigma_{S}$; moreover, its area is close to that of the $P B_{F}$. 


\subsection{An inactivation model: the double-Weibull model}

\subsubsection{Definition}

The analytic form of this model (the deterministic part) proposed by Coroller (2006), including five parameters and one explanatory factor, is:

$$
\eta\left(\theta^{*}, t\right)=C-\log _{10}\left(1+10^{\alpha}\right)+\log _{10}\left[10^{-\left(\frac{t}{\delta_{1}}\right)^{p}+\alpha}+10^{-\left(\frac{t}{\delta_{2}}\right)^{p}}\right]
$$

where $C=\log _{10} N_{0}$ (where $N_{0}$ is the initial number of bacteria), $\alpha$ and $p$ are shape parameters, $\delta_{1}$ and $\delta_{2}$ are treatment times for the first decimal reductions, $\eta\left(\theta^{*}, t\right)$ is the bacteria number (in log units), and $t$ is the time (in hours), the explanatory factor. The vectorial parameter we are interested in is $\theta=\left(C, \alpha, p, \delta_{1}, \delta_{2}\right)^{T}$.

\subsubsection{Results with simulated data}

We first present an illustration with the a priori values $C_{0}=5.45 ; \alpha_{0}=3.15$; $p_{0}=3.67 ; \delta_{10}=5.45 ; \delta_{20}=17.21$, and $\Theta=[5 ; 6]_{C_{00}} \times[2 ; 4]_{\alpha_{0}} \times[1.5 ; 6]_{p_{0}} \times$ $[4 ; 7]_{\delta_{20}} \times[12 ; 20]_{\delta_{20}}$, and $\Xi=[0 ; 20]$ (Coroller, 2006). Using this information we simulated 20 Gaussian $y_{i}$ values (with standard deviation equal to 0.1 ) at 20 equidistant levels of time. We obtained Fig. 3 after computation of $\hat{\theta}=$ $(5.56 ; 3.15 ; 2.99 ; 5.17 ; 16.50)^{T}$ by means of the NLIN procedure of SAS/STAT software. 
For a more relevant comparison, we simulated 1000 data sets, each data set being defined as the pairs $\left\{\left(t_{u}, \tilde{y}_{u}\right) ; u=1, \ldots, 10\right\}$ where $t_{u}$ were always equal to the 20 previous levels. We proceded as in Subsection 5.1.2, and we obtained the simulation results given in Table 2 .

\begin{tabular}{|c|c|c|c|c|c|}
\hline Areas & $\min$ & $\max$ & mean & std & cv\% \\
\hline $\mathcal{A}\left(P B_{t}\right)$ & $8 ; 8 ; 19$ & $10 ; 13 ; 35$ & $9 ; 10 ; 26$ & $0.5 ; 0.96 ; 3.7$ & $5 ; 9 ; 14$ \\
\hline $\mathcal{A}\left(P B_{F}\right)$ & $15 ; 15 ; 33$ & $19 ; 22 ; 62$ & $17 ; 19 ; 47$ & $0.89 ; 1.7 ; 6.6$ & $5 ; 9 ; 14$ \\
\hline $\mathcal{A}\left(P B_{B}\right)$ & $10 ; 11 ; 29$ & $26 ; 42 ; 96$ & $20 ; 32 ; 73$ & $4 ; 8 ; 13$ & $20 ; 24 ; 18$ \\
\hline $\mathcal{A}\left(P B_{X}\right)$ & $31 ; 31 ; 36$ & $33 ; 33 ; 35$ & $32 ; 32 ; 31$ & $0.36 ; 0.54 ; 1.95$ & $1.1 ; 1.7 ; 6$ \\
\hline
\end{tabular}

Table 2 : Coroller model: Simulation statistics of the band areas. The figures are given sequentially according to $\sigma_{S}=0.05 ; 0.1 ; 0.5$, respectively. The figures have been rounded off. 


\subsubsection{An illustration with real data}

With real data (Coroller, 2006), we obtained Fig. 4 where the estimated model curve is based on $\hat{C}_{0}=5.39 ; \hat{\alpha}=3.06 ; \hat{p}=3.53 ; \hat{\delta}_{1}=5.54 ; \hat{\delta}_{2}=17.44$.

The figure 4 should be approximately placed here

Fig. 4: The four bands for the Coroller model for one real data set (crosses stand for the real data). The values of the band areas are: $\mathcal{A}\left(P B_{t}\right)=9 ; \mathcal{A}\left(P B_{F}\right)=$ $15.78 ; \mathcal{A}\left(P B_{B}\right)=9.33 ; \mathcal{A}\left(P B_{X}\right)=16.54$

\subsubsection{Discussion}

This real application is particularly relevant for demonstrating the usefulness of the $P B_{X}$. Indeed, a crucial point here is to have a realistic PCI at the place where the model begins to break down (after about eight days in Fig. 4). At that time, the microbiologist needs a realistic PCI for the log number of bacteria present in the experiment. In this case, we can observe that $P I_{t}$ and $P I_{B}$ are completely false. We confirm that the $P B_{X}$ is not very sensitive to the simulation $\sigma_{S}$. Its area is slightly larger than that of other bands, but that does not prevent its future use. 


\subsection{Another inactivation model: the Leguerinel et al. model}

\subsubsection{Definition}

The analytic form of this model (the deterministic part) proposed by Leguerinel et al. (2005) for modelling the logarithm of the decreasing number of bacteria, including five parameters and three explanatory factors, is:

$$
\eta(\theta ; t, p H, A w)=5-\left(\frac{t}{\delta \times 10\left[\left(\frac{p H-p H_{o p t}}{Z_{p H}}\right)^{2}+\left(\frac{A w-0.985}{Z_{A w}}\right)^{2}\right]}\right)^{p}
$$

where the vector parameter is $\theta=\left(p, \delta, Z_{p H}, Z_{A w}, p H_{o p t}\right)^{T}$, and the explanatory factors are $t$ (the time in minutes), $p H$, and water activity $A w$. Because no rigourous design has yet to be found, real data are not available at that moment for the modelling of such a model. However, it is of particular importance to run some simulations for comparing the different regions. The a priori values provided by Leguérinel et al. (2005) for the parameters and domains are:

$$
\begin{aligned}
p_{0} & =5 ; \delta_{0}=1.8 ; Z_{p H 0}=2.18 ; Z_{A w 0}=0.092 ; p H_{o p t 0}=6.96 \\
\Theta_{0} & =[4.5 ; 5.5]_{p_{0}} \times[1.6 ; 2]_{\delta_{0}} \times[2 ; 2.5]_{Z_{p H 0}} \times[0.0917 ; 0.0930]_{Z_{A w 0}} \times[6.5 ; 7.2]_{p H_{o p t 0}} \\
\Xi & =[0 ; 5378]_{t} \times[4 ; 9]_{p H} \times[0.9 ; 1]_{A w}
\end{aligned}
$$


314

The 72 simulated values were obtained by forming a 3 -dimensional grid on $\Xi$, with respect for the constraint $\eta() \geq$.1 throughout. Table 3 gives the results based on 100 simulated data sets, obtained with $\sigma_{S}=0.2$.

\begin{tabular}{|c|c|c|c|c|c|}
\hline Volumes & $\min$ & $\max$ & mean & std & cv\% \\
\hline $\mathcal{V}\left(P R_{t}\right)$ & 2.68 & 2.95 & 2.82 & 0.06 & 2.15 \\
\hline $\mathcal{V}\left(P R_{F}\right)$ & 4.55 & 5.01 & 4.79 & 0.10 & 2.14 \\
\hline $\mathcal{V}\left(P R_{X}\right)$ & 36.50 & 38.20 & 37.26 & 0.74 & 2.02 \\
\hline
\end{tabular}

Table 3: Inactivation model: simulation statistics of the band areas for $\sigma_{S}=0.2$. $\mathcal{V}\left(P R_{B}\right)$ do not appear here because they are too time-consuming (one computation of $\mathcal{V}\left(P R_{B}\right)$ alone requires about ten hours, while $\mathcal{V}\left(P R_{t}\right)$ and $\mathcal{V}\left(P R_{F}\right)$ require less than three minutes, and $\mathcal{V}\left(P R_{X}\right)$ require about one hour; all the computations were carried out under the same conditions on a Pentium IV Personal Computer).

\subsubsection{Discussion}

This model leads to a larger volume for $P R_{X}$ but, as in the preceding examples, this region is usable mainly because it is a conservative region. 


\section{Conclusion}

We now have a relevant and a statistically rigorous $P B-$ the $P B_{X}-$ for evaluating the global predictive capability of a nonlinear regression model. Since we were able to observe in the preceding examples that its area is not too large, on the one hand, and keeping in mind that it is a conservative band, on the other hand, we are convinced that the $P B_{X}$ can become a useful tool for the practioner in predictive modelling in food. The two usual bands, $P B_{t}$ and $P B_{F}$, can lead to a weak accuracy and, consequently, can be incorrect, especially when $n$ and $\sigma^{2}$ are large. The $P B_{B}$ is based on a second-order approximation, not an exact basis.

The algorithm proposed in Subsection 4.2 for determining the $P B_{X}$ is easy to code and quick to run. Even with five parameters and three explanatory factors in the model (example of Subsection 5.3), less than one hour is necessary on a Pentium IV Personal Computer. The $P B_{B}$ is too time-consuming, even for $p=3$.

The $P B_{X}$ could be effectively used for the comparison of two models by considering the eventual overlapping of their bands. If the two bands overlap, then we will assume that these two models are not significantly different for fitting the data collected, and different if no overlapping exists at all. The intermediate case where overlapping is partial obviously remains a difficult case to resolve. More research is needed in this situation. Finally, a complementary study must be carried out 
when the variance is heteroscedactic. This work is in progress.

Acknowledgements: We would like to express our gratitude to the following institutions, members of the SYMPREVIUS Research Group: ADRIA-Quimper, LUMAQ, INRA, Soredab, CTSCCV, ENVA, AERIAL, for providing data and submitting this problem of prediction bands in predictive modelling in foods.

\section{$7 \quad$ References}

Baranyi, J., Pin, C., Ross, T. (1999). Validating and comparing predictive models. International Journal of Food Microbiology 48: 159-166.

Baranyi, J., Roberts, T.A. (1994). A dynamic approach to predicting bacterial growth in food. International Journal of Food Microbiology 23: 277-294.

Baranyi, J., Roberts, T.A. (1995). Mathematics of predictive food microbiology. International Journal of Food Microbiology 26: 199-218.

Bates, D.M., Watts, D.G. (1988). Nonlinear regression analysis and its applications. John Wiley \& Sons, New York.

Coroller, L. (2006). General model based on two mixed Weibull distributions of bacterial resistance for describing various shapes of inactivation curves. Applied and Environmental Microbiology 72: 10, 6493-6502.

Draper, N.R., Smith, H. (1981). Applied regression analysis. Second Edition. Wiley, New-York. 
Efron, B., Tibshirani, R.J. (1993). An introduction to the bootstrap. Chapman \& Hall.

Gallant, A.R. (1987). Nonlinear statistical models. Wiley \& sons.

Huet, S., Bouvier, A., Poursat, M.-A., Jolivet E. (2004). Statistical Tools for Nonlinear Regression. A Practical Guide with S-PLUS and R Examples. Second Edition. Springer Series in Statistics

Khorasani, F., Milliken, G.A. (1982). Simultaneous confidence bands for nonlinear regression models. Commun. Statist.-Theor. Meth. 11: 11, 1241-1253.

Leguérinel, I., Spegagne, I., Couvert, O., Gaillard, S., Mafart, P. (2005). Validation of an overall model describing the effect of three environmental factors on the apparent D-value of Bacillus cereus spores. International Journal of Food Microbiology 100: 1-3, 223-229.

Oscar, T.P. (2005). Validation of Lag Time and Growth Rate Models for Salmonella Typhimurium: Acceptable Prediction Zone Method. Journal of Food Science 70: 129-137.

Ross, T. (1996). Indices for performance evaluation of predictive. References models in food microbiology. Journal of Applied Bacteriology 81: 501-508.

SAS/IML and SAS/STAT Software, Version 8.1. SAS Institute. Cary. North Carolina. USA.

Vila, J.-P., Gauchi, J.-P. (2007). Optimal designs based on exact confidence regions 
383 for parameter estimation of a nonlinear regression model. J. of Statistical Planning and Inference 137: 9, 2935-2953.

385 Working, H., Hotelling, H. (1929). Applications of the theory of error to the ${ }_{386}$ interpretation of trends. Journal of the American Statistical Association 24: 73387 85. 
Fig. 1: The four bands for the Baranyi-Roberts model for one simulated data set (crosses stand for the simulated data). The values of the band areas (calculated with procedures indicated at the end of Subsection 3.1) are: $\mathcal{A}\left(P B_{t}\right)=$ $21 ; \mathcal{A}\left(P B_{F}\right)=36 ; \mathcal{A}\left(P B_{B}\right)=24 ; \mathcal{A}\left(P B_{X}\right)=42$.

Fig. 2: The four bands for the Baranyi-Roberts model for one real data set (crosses stand for the real data). The values of the band areas are: $\mathcal{A}\left(P B_{t}\right)=70$; $\mathcal{A}\left(P B_{F}\right)=111 ; \mathcal{A}\left(P B_{B}\right)=98 ; \mathcal{A}\left(P B_{X}\right)=166$.

Fig. 3. The four bands for the Coroller model for one simulated data set (crosses stand for the simulated data). The values of the band areas are: $\mathcal{A}\left(P B_{t}\right)=7.82 ; \mathcal{A}\left(P B_{F}\right)=13.97 ; \mathcal{A}\left(P B_{B}\right)=8.90 ; \mathcal{A}\left(P B_{X}\right)=13.38$. It should be noted that days appear on the abscisses, but the parameters are given in the text for the time in hours.

Fig. 4: The four bands for the Coroller model for one real data set (crosses stand for the real data). The values of the band areas are: $\mathcal{A}\left(P B_{t}\right)=9 ; \mathcal{A}\left(P B_{F}\right)=$ $15.78 ; \mathcal{A}\left(P B_{B}\right)=9.33 ; \mathcal{A}\left(P B_{X}\right)=16.54$. 


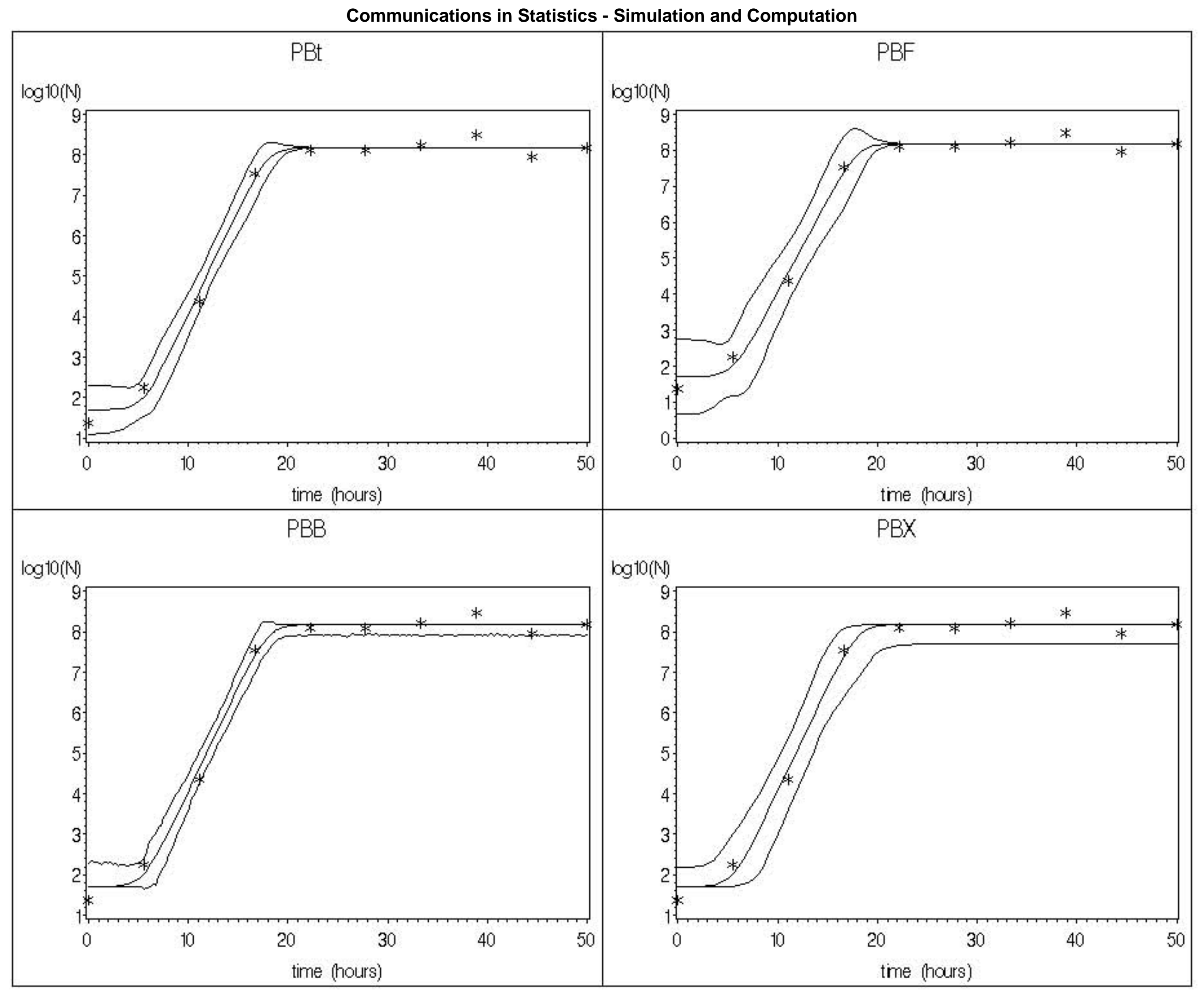

Page 28 of 30

URL: http://mc.manuscriptcentral.com/Issp E-mail: comstat@univmail.cis.mcmaster.ca 


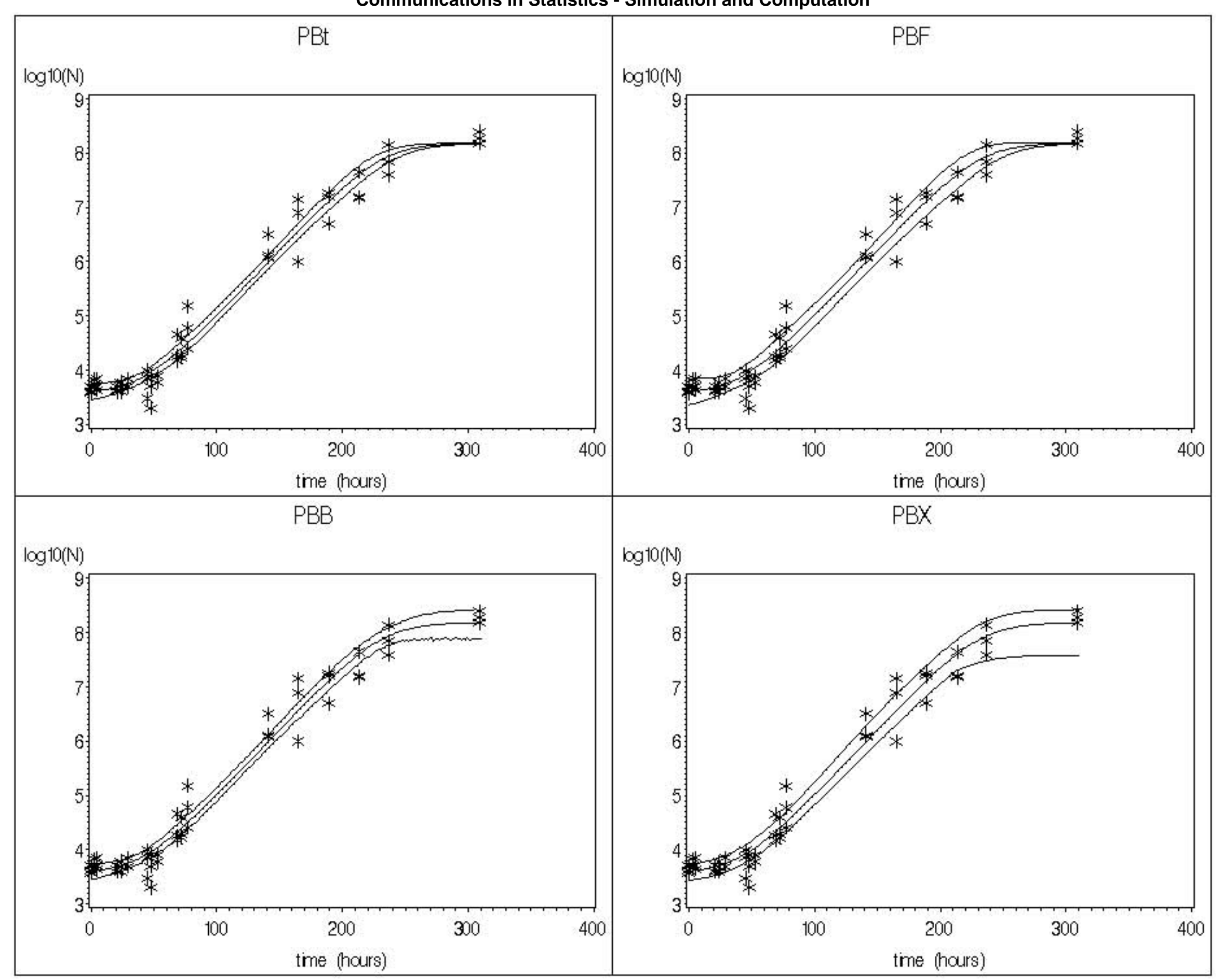

URL: http://mc.manuscriptcentral.com/Issp E-mail: comstat@univmail.cis.mcmaster.ca 


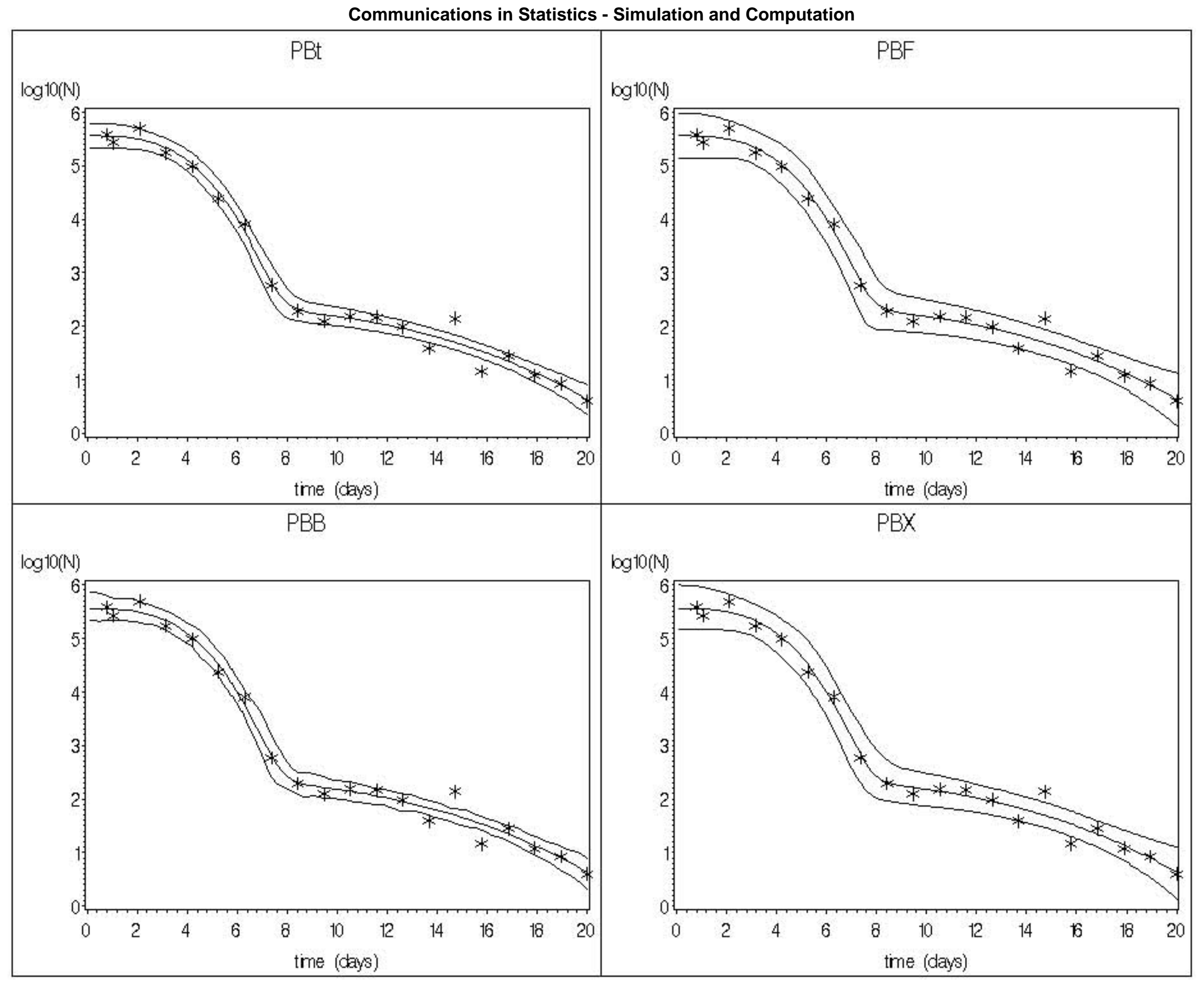




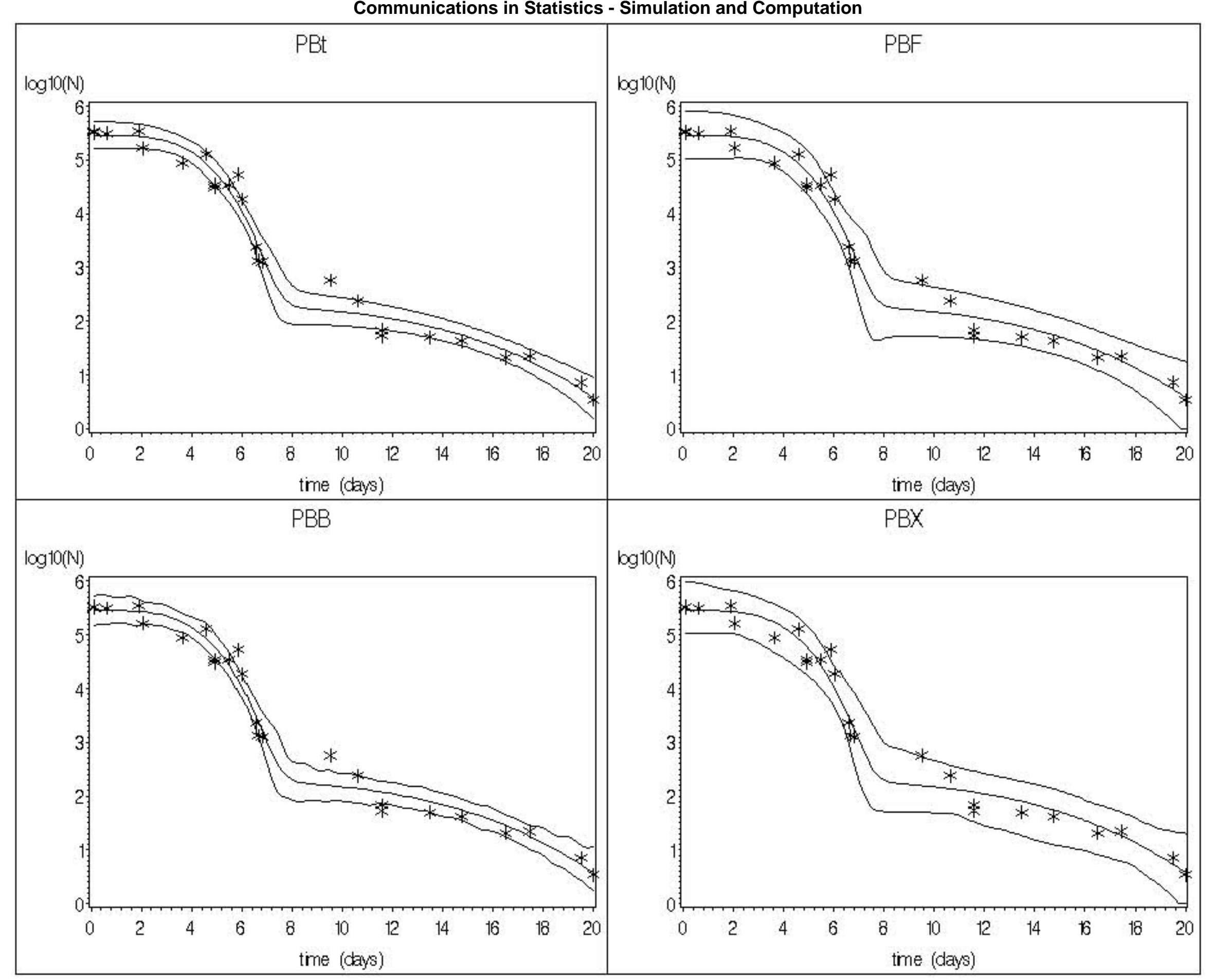

URL: http://mc.manuscriptcentral.com/Issp E-mail: comstat@univmail.cis.mcmaster.ca 